\title{
Current Status of Biportal Endoscopic Decompression for Lumbar Central Stenosis
}

\author{
Bang-Sang Hahn, Jae-Won Jang, Dong-Geun Lee, Choon-Keun Park \\ Department of Neurosurgery, Suwon Leon Wiltse Memorial Hospital, Suwon, Republic of Korea
}

Corresponding Author: Jae-Won Jang, MD

Department of Neurosurgery, Leon Wiltse Memorial Hospital, 437, Gyeongsu-dearo, Paldal-gu, Suwon-si, Gyeonggi-do 16480, Republic of Korea Tel: $+82-10-2794-0747$

Fax: +82-31-223-2322

E-mail: genius60@hanmail.net
Received: February 01, 2021

Revised: February 22, 2021

Accepted: March 05, 2021

\begin{abstract}
Degenerative lumbar spinal stenosis commonly occurs in elderly patients aged above 50-60 years. Surgical intervention is indicated for patient refractory to conservative management, and microscopic decompression has been used for direct spinal canal decompression. The development of surgical instruments and spinal endoscopic system can make possible endoscopic surgery as the useful treatment option for degenerative lumbar disease. Endoscopic spine surgery was mainly preformed in lumbar disc disease through transforaminal route. And then, endoscopic interlaminar approach was introduced using endoscopic drill, more developed endoscopic disc forceps, and Kerrison punches, and so on. Uniportal endoscopic spine surgery through interlaminar space can make possible direct spinal canal decompression in cases with lumbar spinal stenosis, however stiff learning curve still remains the limitation of this surgery. Biportal endoscopic spine surgery was also introduced as the minimal invasive spine surgery, which is basically similar to microscopic surgery and relatively familiar approach to spine surgeons. Biportal endoscopic spine surgery can offer clean operative view in monitor through continuous irrigation and endoscope with large diameter compared to uniportal endoscopic equipment, and this approach may be alternative surgical approach for decompression of lumbar spinal stenosis. Now many spine surgeons perform biportal endoscopic surgery for lumbar central spinal stenosis, and the reports have been published. He we write the technical procedure of biportal endoscopic surgery for lumbar spinal stenosis, discussing about surgical considerations, and also review the surgical outcomes from the previously published articles.
\end{abstract}

Key Words: Biportal, Endoscopy, Lumbar, Central stenosis

\section{INTRODUCTION}

Lumbar central spinal stenosis is one of most common causes of low back, radiating leg pain, and leg claudication. Initial treatment offered is conservative which includes rest, pain medications, physical therapy, or lumbar epidural blocks. Open or microscopic decompressive laminectomy had been performed as the standard surgical procedure for lumbar central stenosis disc which does not improve to conservative management ${ }^{7)}$. Open laminectomy can provide broad decompression of central spinal canal and this surgical procedure is technically familiar and easy to spine surgeons. However extensive injuries of normal spinal structure such as bone, ligaments and paraspinal muscles are inevitable, especially in subtotal laminectomy ${ }^{2)}$.

Beyond the classical open surgical technique, minimally invasive surgical techniques such as mini-open and tubular approaches have been applied to decompression for lumbar central stenosis ${ }^{1422}$. Among them, unilateral laminectomy and bilateral decompression (ULBD) with or without tubular retractor was introduced and has been performed for decompression of central spinal canal, but it also has also some demerits like paraspinal muscle injury, violation of facet joint, and technical difficulty for contralateral decompression ${ }^{6)}$.

In early days, percutaneous endoscopic lumbar discectomy (PELD) via transforaminal route was main stream of endoscopic spine surgery. At that time, disc herniation combined with central spinal stenosis was considered as a contraindication of endoscopic spine surgery in South Korea. However, after the introduction of uniportal interlaminar approach for disc herniation, endoscopic spine surgery has grown by leaps and bounds in the last decade ${ }^{15)}$. With better visualized endoscopes and instruments including endoscopic drills, probes and forceps, etc., now it is possible to treat even central spinal stenosis (not just disc herniation) by spinal endoscopy. Uniportal endoscopic spine surgery is theoretically true and full endoscopic surgery, but endoscopic handling and instruments are not familiar to most of spine surgeons, and it is well known limitation of uniportal endoscopic 
surgery with stiff learning curve ${ }^{1)}$.

Despite the initial report of biportal arthroscopy for lumbar spine by Dr. De Antoni, this approach was not become the main stream of spine surgery during long-time. Recently various biportal spinal endoscopic approaches have been attempted by spine surgeons, especially in South Korea, ${ }^{40,17)}$. With the help of clear magnified surgical view and usage of surgical equipment familiar to most of the spine surgeons, biportal endoscopic surgeons can achieve precise and sufficient decompression from ipsilateral, through midline, to contralateral lateral recess and even contralateral exiting nerve root. Recent reports presented comparable clinical outcomes or even benefits including less muscle damage, and less post-operative opioids usage compared to microscopic decompression.

In this review article, authors will introduce the current useful biportal technical options for lumbar central decompression, and also discuss about the results of previous clinical trials.

\section{SURGICAL PROCEDURES}

Biportal endoscopic decompression for lumbar central stenosis can be divided to unilateral decompression via ipsilateral approach, unilateral decompression via contralateral approach, and ULBD. Most of lumbar central stenosis is developed bilaterally, the ULBD using bi-portal spinal endoscopy will be written in this article.

\section{Instruments Prepare}

Radiolucent frame for prone position, 4-mm arthroscopy or spinal endoscopy, radiofrequency (RF) for bleeding control and soft tissue dissection, endoscopic drill, shaver, and general spinal instrument such as Kerrison punches, disc forceps, and root retractor, nerve freer, and endoscopic hook, etc. Special tool-kit for making endoscopic and working portal will be required.

\section{Anesthesia and Surgical Position}

General or epidural anesthesia can be possible to perform bi-portal endoscopic spine surgery. General anesthesia is recommended to beginner, because muscle relaxation during general anesthesia may be helpful to maintain fluid passage through working portal. It can make clean operation field during operation via wash out of blood and bone dust. After general anesthesia, the patient is placed in the prone position over the radiolucent spine frame in a flexed position.

\section{Skin Entry Points and making working and Endos- copic Port}

The target level for decompression was localized by fluoroscopy. Medial pedicular line of target level is marked on true anteroposterior fluoroscopic view. Two transverse or vertical incision about within $1 \mathrm{~cm}$ are made on meeting point between medial pedicular line and distal pedicle line of upper and lower vertebra on true fluoroscopic lateral view, respectively. If the surgeon is right-dominant hand, spinal endoscopy is handled by left hand and spinal instruments including Kerrison punches, $\mathrm{RF}$, and drill are operated by right hand. In order to make abundant outflow of irrigation fluid, linear or cruciate fascial incision using a 15-blade scalpel and serial dilation should be done. Multifidus muscle can be easily separated by serial dilation and blunt dissection using freer or muscle serial dilator. During the serial dilation, docking point of spinal endoscopy and serial dilator is spino-laminar junction of targeted lamina. Because fatty space between multifidus and lamina, the surgeon can make working space reducing normal structure destruction. Isotonic saline should be used to prevent water intoxication, and continuous abundant irrigation should be also maintained. Sometimes water pump can be used to maintain water irrigation, but the authors prefer natural drainage of irrigation fluid via working port (Figure 1).

\section{Decompressive Laminectomy and Ipsilateral Decom- pression}

After RF ablation of remaining soft tissue between lamina and ligamentum flavum, laminectomy is initiated on spino-laminar junction of target level. Ipsilateral laminotomy is done using endoscopic drill, small osteotome, and Kerrison punches under clean magnified endoscopic view. After identification of central
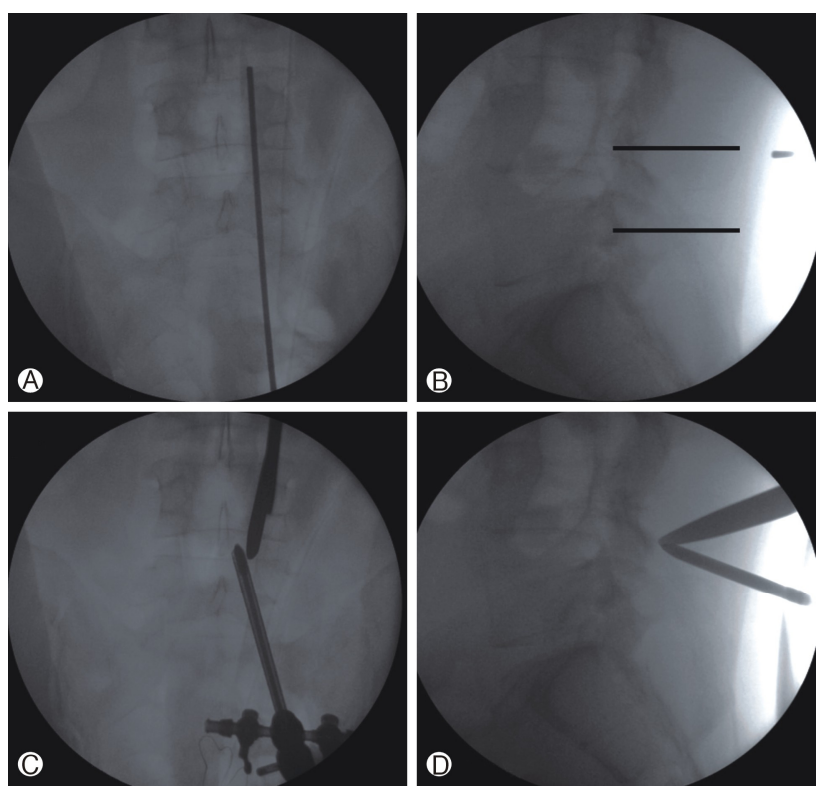

Figure 1. Two portal entry point and making hole. (A): Skin incision sites were decided around pedicle area. Medial pedicular line of approach site on lesion level was firstly drawn in the trun anteroposterior simple radiograph. (B): Two skin incision were made on medial pedicular line around mid to lower margin of proximal and distal pedicle of lesion level, separated by $2-3 \mathrm{~cm}$ in the true lateral simple radiograph. (C) and (D): After serial dilation of port for endoscopy and working channel, surgeon can fight instruments with triangular formation through each port on spino-laminar junction, initial target area of operation. 
fissure of ligament flavum, ipsilateral laminotomy is performed till medial portion of superior articular process is exposed. The proximal origin of ligamentum flavum is Y-shaped and inserts cranio-laterally up to neuroforamen, therefore laminectomy should be extended more cranially on the lateral border when till the flavum edge is freed. Outer part of ligamentum flavum is attached on the upper portion of lamina of lower vertebra, therefore distal part of outer ligamentum flavum can be easily detached from upper portion of lamina using angled curettes. After sufficient bony resection and removal of outer layer of ligamentum flavum, inner layer of ligamentum flavum is removed. If there is severe lateral recess stenosis, the author prefers to use small osteotome instead of Kerrison punch to prevent dura injury and nerve root damage. Ideal decompression should be performed to medial margin of pedicle of lower vertebra. Finally, epidural ligaments between nerve root and ventral dura surface should also be removed to release neural structure adhesion.

\section{Contralateral Sublaminar Decompression}

Ligamentum flavum of contralateral side can be easily detached from ventral side of opposite lamina using freer or curettes. As the ipsilateral decompression, contralateral sublaminoplasty should be extended more cranially on the lateral border when till the flavum edge is freed. The head of endoscopic drill should be used the space between lamina and dorsal surface of ligamentum flavum to prevent unintended dura or neural structure injuries. Sublaminoplasty should be extended till medial border of superior articular process is exposed. Ligamentum flavum is removed and lateral recess can be fully decompressed using curettes, angled disc forceps, small osteotomes, or Kerrison punches. Epidural adhesion between nerve root and ventral dura surface is released using angled hooks. The author confirms medial border of contralateral pedicle (Figure 2).
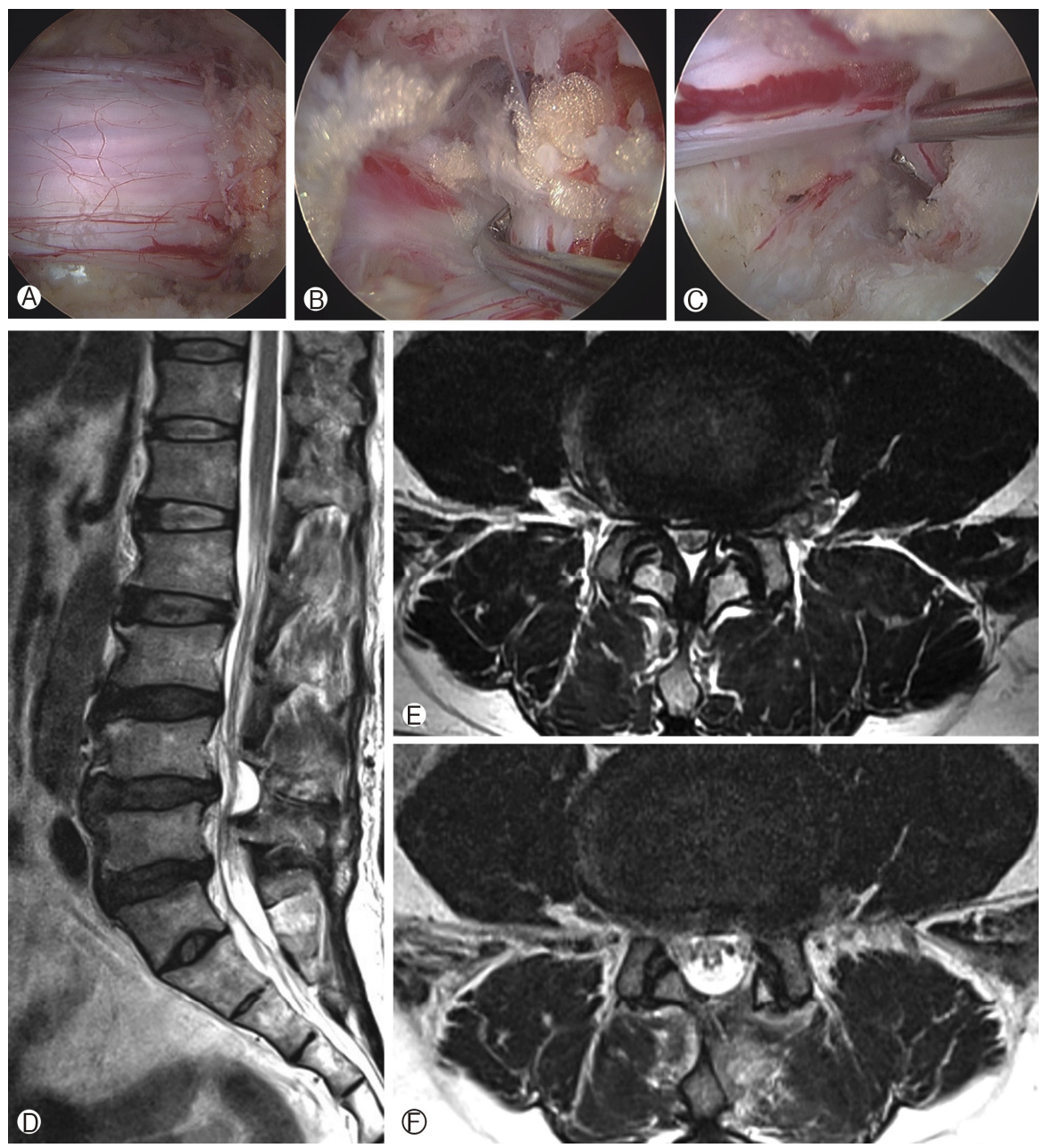

Figure 2. (A)-C): Intraoperative endoscopic photographs after biportal endoscopic decompression for lumbar central stenosis. Central spinal canal was well decompressed with lateral $2-3 \mathrm{~mm}$ free margin and both traversing nerve root were also well decompressed and medially released with the showing the medical pedicle margin. Preoperative $(\mathbb{D}, \mathbb{E})$ and postoperative $(\mathbb{E}),(\mathrm{G})$ ) magnetic resonance T2-weighted images showed well decompression of stenotic lesion at L4-5 level. 


\section{Bleeding Control and Closure}

Epidural bleeding is easily controlled by RF ablation. Exposed cancellous bone should be sealed using bone wax to prevent delayed oozing after stopping of saline irrigation. A drainage catheter is inserted through the working port with stay suture to prevent of postoperative epidural hematoma. Subcutaneous layers are approximated with absorbable suture material and skin layers are closed with non-absorbable material or skin tape.

\section{REVIEWS OF OUTCOMES AND COMPLICATIONS}

Although the initial report of biportal spinal endoscopy for lumbar spine was firstly described by Dr. De Antoni, however active clinical trial and expansion of biportal endoscopic spine surgery have been performed by Korean spinal surgeons ${ }^{4,10,18)}$. Total 21 articles were found in Pubmed when we searched the title name; unilateral biportal spinal endoscopy and central spinal stenosis. Among them, several important article will be reviewed on following sentence to clarify the effectiveness of biportal endoscopy and complications.

Eum et al. firstly described their preliminary clinical result of biportal endoscopy for the treatment of lumbar spinal stenosis in $2016^{5)}$. In this article, he introduced the surgical procedure of biportal endoscopy and relatively favorable clinical outcomes. Among total 58 patients, 47 patients were excellent or good result according to the Macnab criteria and Oswestry Disability Index (ODI) was also improved from 67.2 to 24.3 at 6 months after surgery. Complications were developed in 8 cases; 3 postop headache, 2 durotomy, 2 postoperative numbness, and 1 postoperative epidural hematoma. Despite early trial of biportal endoscopy to lumbar central spinal stenosis, successful results were reported without serious complications.

Prospective case-control study between biportal endoscopic decompression and microscopic decompression was reported by Dr Heo and Park ${ }^{11)}$. Postoperative dura expansion, improvement of ODI score, and visual analogue scale score were all improved in both two group. Only visual analogue scale (VAS) score of back pain at one day after surgery was significantly greater in the microscope group than in the endoscopy group. Complications were durotomy and epidural hematoma, however there were no difference of incidence between microscopic and endoscopic groups. Min et al. also reported similar results through their comparative study. There was a significant difference between endoscopic and microscopic groups VAS of back on postoperative 2 months. VAS of back was significantly greater in microscopic group ${ }^{16)}$.

Park et al. reported that hospital stay, postoperative pain and fentanyl usage for pain control were significantly lower in the biportal endoscopy group compared to the microscopic group in their randomized controlled trial study ${ }^{18)}$. In the follow-up results, there were no significant differences of functional outcomes such as ODI, European Quality of Life-5 Dimensions (EQ-5D) scores between the biportal endoscopy and microscopic groups. The rate of complications was also similar, therefore they concluded that biportal endoscopic decompression for lumbar spinal stenosis showed noninferiority compared to microscopic decompression $^{19)}$.

Last review article is about comparative study of three technique; uniportal endoscopy, biportal endoscopy, and microscopic decompression for the treatment in the lumbar canal stenosis. Although mean dural expansion was significantly lower in uniportal endoscopic group than that of biportal endoscopic group and microscopic group, clinical outcomes were similar in all three groups. Immediately pain recovery showed superiority in uniportal and biportal endoscopic groups compared to microscopic group. The mean angle of facetectomy area in the biportal endoscopy was significantly lower than that in the uniportal endoscopic group and microscopic group ${ }^{10)}$.

As the results of published reviewed article, biportal endoscopic decompression for the treatment of lumbar central spinal stenosis showed favorable radiologic and clinical outcomes without significantly newly developed complications compared to conventional surgery.

\section{DISCUSSION}

Conventional decompresive laminectomy had been performed as the surgical option in patients with symptomatic lumbar central stenosis ${ }^{2,7)}$. However, injury of normal tissue for canal decompression has been remained the matter of surgical limits. Developments of operative microscopy and instruments had enabled bilateral spinal canal decompression through unilateral laminotomy, a less invasive approach to treat central stenosis ${ }^{6}$. More recently in last two decades, endoscopic spine surgery as more minimal invasive technique compared to microscopic surgery has been performed actively ${ }^{15}$. As one of minimally invasive surgery for lumbar central stenosis, biportal endoscopic decompression not only can result in less injury to the patients (small skin incision, less muscle injury, less facet joint injury and blood loss, etc.), but also can achieve a sufficient decompression. These features affected to various clinical parameters and incidence of complication, ultimately to patient satisfaction.

Compared to uniportal endoscopic decompression, Heo et al. reported that biportal endoscopic technique achieved significantly more dural expansion at final follow up (323.4 469.7 $\mathrm{mm}^{2}$ in biportal versus $260.7 \pm 45.5 \mathrm{~mm}^{2}$ in uniportal) ${ }^{10)}$. The mean angle of facetectomy was significantly lower in bi-portal endoscopic decompression group. There were no significant differences in clinical results such as leg and back VAS, ODI between two approaches. Preservation of facet joint after decompressive laminectomy may be very important to maintain spinal stability, because extensive removal of the lamina and violation of facet joint leading to iatrogenic instability, which may require a fusion surgery ${ }^{8}$. Laminectomy can be performed effectively without significantly loss of facet joint area in biportal endoscopic surgery, because angled curettes, angled Kerrison punch, and endoscopic drill are freely used through movable working port and clean sur- 
gical view. This advantage in biportal endoscopy will be important point to obtain the favorable outcomes and maintain spinal stability during long-term follow up.

Several complications after biportal endoscopic spine surgery may be developed. Because of limited monitor view of whole surgical field, beginners may experience poor anatomical orientations, which occasionally results in an excessive facetectomy, iatrogenic facet fracture or imperfect decompression". For a beginner, it is crucial to have a detailed comprehension of anatomic structures of lumbar spine through open microscopic surgery and always get a supervision by well experienced endoscopic surgeon. Current monitor view of endoscopy is 2-Dimentional image and it makes high risk of durotomy or neural tissue injury during endoscopic drilling or other decompressive procedures. The authors recommend that last bone work using endoscopic drill should be completed before complete removal of ligamentum flavum to reduce the risk of durotomy or neural tissue injury. Fortunately, ligamentum flavum can be separated as the two layers; inner and outer layer. Surgeons can effectively remove outer layer of ligamentum flavum using angled curettes, and last bone work using endoscopic drilling can be performed completely under preservation of inner layer of ligamentum flavum. Further bone work using small osteotomes or angled Kerrison punch may be useful after complete removal of ligamentum flavum. To prevent the durotomy or root injury, maintenance of clean surgical field through abundant saline irrigation is also very important. Previously published study reported the occurrence of postoperative epidural hematoma ${ }^{5,18)}$. Continuous saline irrigation offers clean surgical field and hydrostatic pressure on epidural venous flexus and exposed cancellous bone also provides effect of bleeding control during surgical procedures. However, bleeding may be occurred after cessation of continuous saline irrigation and hydrostatic pressure effect on working space. Bleeding point on epidural space and exposed cancellous bone should be checked and controlled on endoscopic view after continuous irrigation. Lastly, the authors recommend the inser-

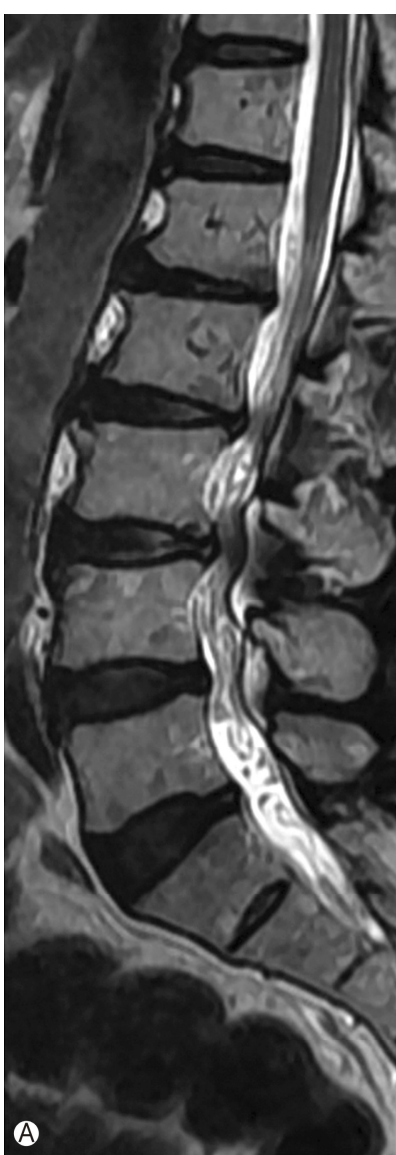

(A)

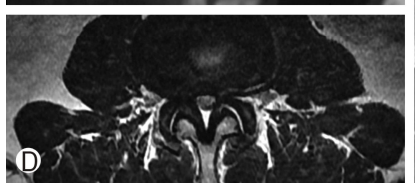

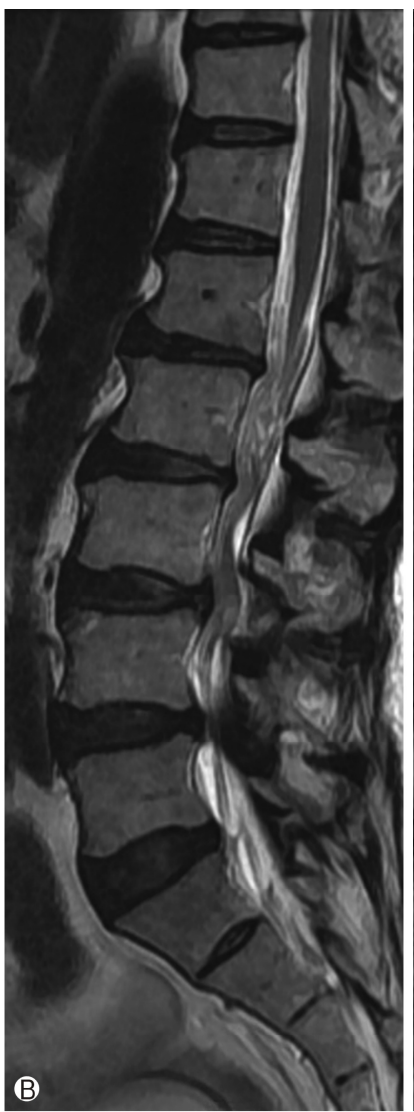
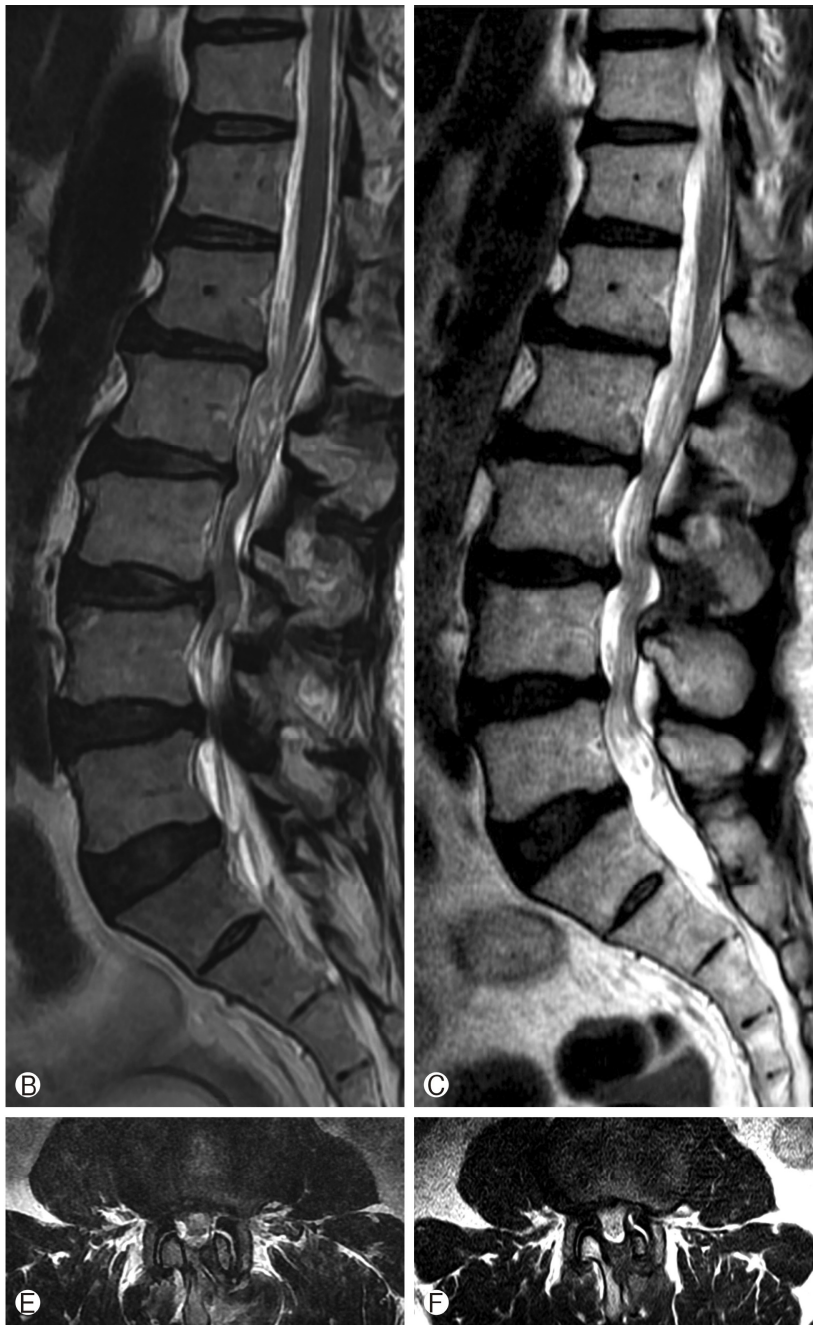

Figure 3. Preoperative (A), (B)), Postoperative (B), (E)), and 6 months after surgery (C), ()) magnetic resonance T2-weighted images showed insufficient decompression of central and lateral recess stenosis and early development of restenosis at L3-4 level. 
tion of drainage catheter on epidural space without root irritation to prevent epidural hematoma. Understand and management of the hydrostatic pressure during endoscopic surgery is very important. Surgeons can take advantage of hydrostatic pressure for controlling the bleedings from epidural vessels and oozing from exposed cancellous bones. Also, it helps to push the dura mater to the bottom and make working space, being especially helpful during decompression of contra-lateral side lateral recess in terms of obtaining sufficient view of surgical field. On the other hand, too much hydrostatic pressures by uncontrolled irrigation pump or improper outflow can cause elevation of intracranial pressure, which leads to headache, more seriously seizure after the surgery. Hydrostatic pressure of about $30 \mathrm{mmHg}$ under maintaining continuous fluent outflow is thought to be safe in lumbar endoscopic surgeries, because most of oozing from epidural space and bone can be controlled under $25 \mathrm{mmHg}$ hydrostatic pressure ${ }^{3,12}$. The authors prefer natural maintenance of outflow without pressure pump. Usage of semicircular tube in working port is helpful the maintenance of abundant outflow. Saline back is located with the height of $40-50 \mathrm{~cm}$ from working area, because hydrostatic pressure is made $22 \mathrm{mmHg}$ per $30 \mathrm{~cm}$ gap of height between two points ${ }^{13)}$.

Adequate decompression can offer long-term favorable outcome, so it is important to check proper surgical endpoint of decompression. Firstly, decompression is recommended over 2-3 mm laterally from dura lateral margin on disc level, because dura is shrunk by hydrostatic pressure during operation and true lateral margin of dura may be located more laterally under natural state. It may be also helpful the prevention of restenosis by preserved spinal motion. Secondly, the authors always confirmed medial margin of pedicle on lateral recess level, and it made traversing nerve root freely without compression. Lastly, epidural adhesion between ventral dural surface and dura including nerve root should be released using angled hook. Nerve root and dura could be easily retracted medially without resistance after removal of adhesion, and it may be valuable to reach favorable outcome. If the sufficient decompression was not performed including lateral recess, early restenosis may be developed (Figure 3).

Two similar articles of systematic review and meta-analysis concluded that bi-portal endoscopic technique could be considered as an alternative to the microscopic decompression ${ }^{20,211}$. There were no significant differences in VAS score for leg and back pain, ODI at final follow-up between bi-portal endoscopic decompression and microscopic decompression. The mean operation time and complication rate were similar in both surgical approaches. Bi-portal endoscopic decompression group showed potential benefits in terms of less opioid usage, early ambulation and shorter hospital stay.

Published article showed favorable outcome after biportal endoscopic decompression for lumbar spine stenosis. However, most of study technical reports with preliminary surgical results or retrospective comparative study. Although single randomized control trial study published recently, this study has some limitations such as small number of cases, short follow-up period, and single center study. In the future, multicenter randomized trial with large number and long-term follow-up period will be mandatory to clarify the advantages of biportal endoscopic decompression for the treatment of lumbar spinal stenosis.

\section{CONCLUSIONS}

Bi-portal endoscopic decompression seems to be a feasible treatment option for lumbar central stenosis with clean operative view. Although lack of large scaled and long term followed up data yet, it has been showing comparable clinical outcomes and potential benefits over microscopic decompression, especially in rapid postoperative pain recovery. To demonstrate the superiority of biportal endoscopic surgery, many clinical studies including multicenter randomized trial will be required.

\section{CONFLICT OF INTEREST}

No potential conflict of interest relevant to this article.

\section{REFERENCES}

1. Bing Wang, Guohua Lu, Alpesh A Pate, Peigen Ren, Ivan Cheng: An evaluation of the learning curve for a complex surgical technique: The full endoscopic interlaminar approach for lumbar disc herniations. Spine J 11(2):122-130, 2011

2. Celik SE, Celik S, Goksu K, Kara A, Ince I: Micro-decompressive laminotomy with a 5 -year follow-up period for severe lumbar spinal stenosis. J Spinal Disord Tech 23:229-235, 2010

3. Choi CM, Chung JT, Lee SJ, Choi DJ: How I do it? Biportal endoscopic spinal surgery (BESS) for treatment of lumbar spinal stenosis. Acta Neurochir (Wien) 158(3):459-463, 2016

4. Daniel De Antoni, Maria Laura Claro, Gary G. Poehling, Steven S. Hughes: Translaminar Technical Note Lumbar Epidural Endoscopy: Technique, and Indications Anatomy. Arthroscopy 12(3): 330-334, 1996

5. Eum JW, Heo DH, Son SK, Park CK: Percutaneous biportal endoscopic decompression for lumbar spinal stenosis: A technical note and preliminary clinical results. J Neurosurg Spine 24(4):602607, 2016

6. Francesco Costa, Marco Sassi, Andrea Cardia, et al.: Degenerative lumbar spinal stenosis: Analysis of results in a series of 374 patients treated with unilateral laminotomy for bilateral microdecompression. J Neurosurg Spine 7(6):579-586, 2007

7. Gibson JN, Waddell G: Surgery for degenerative lumbar spondylosis. Cochrane Database Syst Rev 4:CD001352, 2005

8. Guha D, Heary RF, Shamii MF: Iatrogenic spondylolisthesis following laminectomy for degenerative lumbar stenosis: Systematic review and current concepts. Neurosurg Focus 39:E9, 2015

9. Hahn BS, Park JY: Incorporating New Technologies to Overcome the Limitations of Endoscopic Spine Surgery: Navigation, Robotics, and Visualization. World Neurosurg 145:712-721, 2020

10. Heo DH, Lee DC, Park CK: Comparative analysis of three types of minimally invasive decompressive surgery for lumbar central stenosis: Biportal endoscopy, uniportal endoscopy, and microsurgery. Neurosurg Focus 46(5):E9, 2019

11. Heo DH, Quillo-Olvera J, Park CK: Can Percutaneous Biportal 
Endoscopic Surgery Achieve Enough Canal Decompression for Degenerative Lumbar Stenosis? Prospective Case-Control Study. World Neurosurg 120:e684-2689, 2018

12. Kang MS, Park HJ, Hwang JH, Kim JE, Choi DJ, Chung HJ: Safety Evaluation of Biportal Endoscopic Lumbar Discectomy: Assessment of Cervical Epidural Pressure During Surgery. Spine (Phila Pa 1976) 45(20):E1349-E1356, 2020

13. Kang T, Park SY, Lee SH, Park JH, Suh SW: Assessing changes in cervical epidural pressure during biportal endoscopic lumbar discectomy. J Neurosurg Spine 30:1-7, 2020

14. Khoo Lt, Fessler RG: Microendoscopic decompressive laminotomy for the treatment of lumbar stenosis. Neurosurgery 52:S146S154, 2002

15. Kim M, Kim HS, Oh SW, et al.: Evolution of spinal endoscopic surgery. Neurospine 16:6-14, 2019

16. Min WK, Kim JE, Choi DJ, Park EJ, Heo J: Clinical and radiological outcomes between biportal endoscopic decompression and microscopic decompression in lumbar spinal stenosis. J Orthop Sci 25(3):371-378, 2020

17. Pao JL, Lin SM, Chen WC, Chang CH: Unilateral biportal endoscopic decompression for degenerative lumbar canal stenosis. J Spine Surg 6(2):438-446, 2020
18. Park SM, Kim GU, Kim HJ, et al.: Is the Use of a Unilateral Biportal Endoscopic Approach Associated with Rapid Recovery After Lumbar Decompressive Laminectomy? A Preliminary Analysis of a Prospective Randomized Controlled Trial. World Neurosurg 128:e709-e718, 2019

19. Park SM, Park JW, Jang HS, et al.: Biportal endoscopic versus microscopic lumbar decompressive laminectomy in patients with spinal stenosis: A randomized controlled trial. Spine J 20(2):156165, 2020

20. Raymond Pranata, Michael A Lim, Rachel Vania, Julius July: Biportal Endoscopic Spinal Surgery versus Microscopic Decompression for Lumbar Spinal Stenosis: A Systematic Review and Meta-Analysis. World Neurosurg 138:e450-e458, 2020

21. Tiewu Chen, Guoqing Zhou, Zhineng Chen, Xinmiao Yao, Dan Liu: Biportal endoscopic decompression vs. microscopic decompression for lumbar canal stenosis: A systematic review and metaanalysis. Exp Ther Med 20(3):2743-2751, 2020

22. Yasuo Mikami, Masateru Nagae, Takumi Ikeda, Hitoshi Tonomura, Hiroyoshi Fujiwara, Toshikazu Kubo: Tubular surgery with the assistance of endoscopic surgery via midline approach for lumbar spinal canal stenosis: a technical note. Eur Spine J 22(9): 2105-2112, 2013 